Check for updates

Cite this: RSC Adv., 2017, 7, 24291

Received 3rd March 2017 Accepted 25th April 2017

DOI: $10.1039 / \mathrm{c} 7 \mathrm{ra02626a}$

rsc.li/rsc-advances

\title{
A triterpenoidal saponin fraction of Conyza blinii H.Lév. is a dual-targeting autophagy inhibitor for HeLa cells $\dagger$
}

\author{
Haiyan Liu, ${ }^{a}$ Chenxi Hu, ${ }^{a}$ Nana Sun, ${ }^{a}$ Yuyin Li, ${ }^{a}$ Shuli Man, ${ }^{a}$ Zhenxing Liu, ${ }^{a}$ Aipo Diao ${ }^{\text {ab }}$ \\ and Long $\mathrm{Ma}$ (D) *ab
}

\begin{abstract}
Autophagy is a highly conserved cellular process in eukaryotic cells, which allows the orderly degradation and recycling of cellular components via lysosomal pathway. It is believed that autophagy is highly relevant to oncogenes and oncotherapy. Increasing evidence suggests that pharmacological modulation, especially inhibition of autophagy is a promising therapeutic strategy for cancers. In current study, we have demonstrated that the saponins fraction (Conyza blinii saponins, CBS) isolated from a medicinal plant Conyza blinii H.Lév. is a dual-targeting autophagy inhibitor for HeLa cells. The inhibition effect of CBS is derived from its mTOR activation and autophagic degradation blockage effects. Our novel finding not only expands the bio-activity spectrum of saponins but also creates potential for anti-cancer drug development.
\end{abstract}

\section{Introduction}

Over the centuries, people have been fascinated by the immense diversity in both the structures and bio-activities of natural products. ${ }^{1-4}$ Humans have harnessed the wisdom of using them ethno-pharmacologically as therapy against several diseases since ancient times. Nowadays, products originating from nature still have a profound impact on drug discovery in particular for cancers. ${ }^{5,6}$ We have long been interested in a medical plant called Conyza blinii H.Lév., a herbaceous member belonging to Compositae. It is mainly distributed in Sichuan and Yunnan provinces of China and commonly called Jin Long Dan Cao. Its dried overground section is valuable in folk medicine for treatment of chronic bronchitis, gastroenteritis and some other inflammatory diseases. Pharmacological and clinical studies have indicated that Conyza blinii H.Lév. exhibits antibacterial, anti-inflammatory and expectorant effects, which are well recorded in official guidance of traditional Chinese medicine (TCM) such as Pharmacopoeia of the People's Republic of China 2010 edition. ${ }^{7}$ Chemical profiles of this species have revealed many natural products including diterpenoids, flavonoids, triterpenoids and saponins. CBS

${ }^{a}$ Key Laboratory of Industrial Fermentation Microbiology, Ministry of Education, School of Biotechnology, Tianjin University of Science \& Technology, Tianjin 300457, China. E-mail: malong@tust.edu.cn; woshimalong1983@163.com; Fax: +86 22 60602298; Tel: +862260602948

${ }^{b}$ Tianjin Key Laboratory of Industry Microbiology, School of Biotechnology, Tianjin University of Science \& Technology, Tianjin 300457, China

$\dagger$ Electronic supplementary information (ESI) available. See DOI: $10.1039 / \mathrm{c} 7 \mathrm{ra02626a}$
(Conyza blinii saponins) is the oleanane type pentacyclic triterpenoidal saponin fraction of Conyza blinii H.Lév., which is considered as the major bioactive constituents in this plant. CBS sample has been subjected to high resolution HPLCtandem mass spectrometric analysis. ${ }^{8}$ It is found that CBS has a number of triterpenoidal saponins based on retention times and HPLC-MS ${ }^{n}(n=2-4)$ data. Among them, 15 saponins have been previously reported by $\mathrm{Su} e t$ al. ${ }^{9-11}$ Our group have previously confirmed that CBS has a gastric mucous membrane protection activity against acute gastric ulcer induced by ethanol using rodent models. ${ }^{12}$ In 2016, we have discovered that CBS has a strong cytostatic activity against a range of solid tumour cells including HeLa, A549, MGC-803 and MCF-7 cells. Amongst these cells, CBS is most cytotoxic against HeLa cells; a $19.4 \pm 1.94 \mu \mathrm{g} \mathrm{mL} \mathrm{m}^{-1}(24 \mathrm{~h})$ and a $8.56 \pm 1.21 \mu \mathrm{g} \mathrm{mL} \mathrm{m}^{-1}(48 \mathrm{~h})$ $\mathrm{IC}_{50}$ values against HeLa have been reported. ${ }^{13}$ In vivo experiment has confirmed its anti-cancer activity, since it has been observed that $15 \mathrm{mg} \mathrm{kg}^{-1}$ CBS (i.p.) is a tolerable dose and reduces the tumor weight by $70 \%$ in a 10 day administration regimen using xenografted mouse models. Mechanistically, we have further demonstrated that the anti-cancer activity of CBS is partially from its NF- $\kappa \mathrm{B}$ singling pathway inhibition. ${ }^{14}$

Macroautophagy (hereafter referred to as autophagy) is an evolutionarily conserved and catabolic 'self-eating' process, in which certain cytoplasmic components are enclosed within a double-membraned vesicle termed as autophagosome (AP), and subsequently, an AP fuses with a lysosome (LY) to form an autophagolysosome (AL). The contents in AL are degraded enzymatically. Autophagy leads to generation of amino acids, sugars, fatty acids, and nucleosides which are recycled for macromolecular synthesis and energy production. ${ }^{15}$ Increasing 
evidence suggests crucial roles of autophagy in cancers. ${ }^{\mathbf{1 6}}$ Generally speaking, autophagy activation in normal cells may suppress tumourigenesis, whereas its inhibition may be beneficial for the therapy of established tumours. ${ }^{17,18}$ Due to its 'double-edged sword' roles in both oncogenesis and oncotherapy, autophagy has become one of the most attractive therapeutic targets for cancer treatment.

\section{Materials and methods}

\subsection{Chemicals and antibodies}

Rapamycin (RAPA), chloroquine diphosphate salt (CQ) and bafilomycin A1 (BAF), monodansylcadaverine (MDC), acridine orange (AO) and 3-(4,5-dimethylthiazol-2-yl)-2,5-diphenyltetrazoliumbromide (MTT) were obtained from from SigmaAldrich (MO, USA). Cisplatin injection was purchased from Qilu Pharmaceutical Co., LTD (Jinan, China). Commercial antibodies were purchased from the following sources: antibodies against LC3, p62, and phospho-mTOR (Ser 2448) were purchased from Santa Cruz Biotechnology; antibodies against p-p70S6 (p-S6) were from Proteintech Group; antibodies against LAMP1 and LAMP2 were from Abcam. Fluorophore and HRPconjugated secondary antibodies were purchased from Invitrogen.

\subsection{Preparation and analysis of CBS}

A voucher specimen of Conyza blinii H.Lév. was identified by Prof. Tianxiang Li (Tianjin University of Traditional Chinese Medicine, China). Preparation of CBS from dried Conyza blinii H.Lév. was strictly conducted as previously described by its provider Professor Yanfang Su. ${ }^{12}$

\subsection{HPLC-MS analysis}

High resolution HPLC-MS was conducted according to previously publication with slight modifications. ${ }^{8}$ Simply, samples were separated on a COSMOSIL Cholester column equipped with a guard column. The mobile phase consisted of water (A) and acetonitrile (B). A gradient program was used for elution: 0 min, $90 \%$ A, 10\% B; 5 min, 70\% A, 30\% B; $30 \mathrm{~min}, 30 \%$ A, $70 \%$

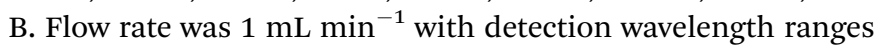
from 190 to $800 \mathrm{~nm}$. The column temperature was set at $30{ }^{\circ} \mathrm{C}$.

\subsection{Cell culture}

HeLa cells were maintained in DMEM medium. A549 cells were grown in F-12K medium supplemented with $10 \%$ fetal bovine serum (FBS), $2 \mathrm{mM}$ glutamine, $100 \mathrm{U} \mathrm{mL}^{-1}$ penicillin and $100 \mathrm{mg} \mathrm{mL} \mathrm{m}^{-1}$ streptomycin. All cells were incubated in a humidified atmosphere at $37{ }^{\circ} \mathrm{C}$ with $5 \% \mathrm{CO}_{2}$ infusion.

\subsection{Fluorescence imaging of cells}

For DAPI staining, HeLa cells $\left(3 \times 10^{5}\right.$ per well) were seeded on the glass coverslips in 6-well plates and were left to adhere overnight. Different concentrations of agents where necessary were added and incubated. After this, the coverslips were washed three times with PBS before fixed in $4 \%$ paraformaldehyde for $20 \mathrm{~min}$. The cells on the coverslips were stained with DAPI for $30 \mathrm{~min}$ in the dark at room temperature, after staining, cells were washed three times with PBS. The coverslips were immediately observed and photographed using a fluorescence microscope (Olympus). For AO staining, HeLa cells $\left(1 \times 10^{5}\right.$ per well) were seeded and cultured at $37^{\circ} \mathrm{C}$ for $24 \mathrm{~h}$. Different concentrations of agents where necessary were added and incubated. After this, $2 \mu \mathrm{M}$ AO or $50 \mu \mathrm{M}$ MDC was added to the cells and incubated at $37^{\circ} \mathrm{C}$ for $15 \mathrm{~min}$ and $40 \mathrm{~min}$ respectively in the dark. Then the cells were washed three times with Dulbecco's phosphate buffered saline (DPBS). Images were captured and analysed using a fluorescence microscopy (Olympus).

\subsection{Observations of autophagic vacuoles (AVs) by transmission electronic microscopy (TEM)}

HeLa cells with or without CBS treatment were fixed in $2.5 \%$ glutaraldehyde ( $\mathrm{pH} 7.5$ ) at $4{ }^{\circ} \mathrm{C}$ for $\sim 2 \mathrm{~h}$ and post-fixed with $0.1 \mathrm{M}$ osmic acid for $3 \mathrm{~h}$ at room temperature. After fixation, cells were dehydrated in a gradient of $50-100 \%$ ethanol and embedded in Spurr resin. $70 \mathrm{~nm}$ ultra-thin sections were obtained using a microtome (Leica EM UC6). After double staining with lead citrate and uranyl acetate, sections were observed and pictured under a transmission electron microscope (JEM-1230; JEOL) at $40-120 \mathrm{kV}$ voltages.

\subsection{Cell lysis and western blots}

For the immunoblot, the cells were placed on ice, washed twice with cold PBS, and lysed in RIPA buffer containing $50 \mathrm{mM}$ Tris$\mathrm{HCl}$ (pH 7.4), $150 \mathrm{mM} \mathrm{NaCl}, 0.5 \mathrm{mM}$ DTT, 2 mM EDTA, and 1\% NP-40 with protease inhibitors. Western blots were carried out by separating the samples using SDS-PAGE gels, and the gels were then transferred to a PVDF membrane for blotting. The concentration of protein sample was assayed using the Bradford method. No high-contrast (overexposure) of gels/blots was used.

\subsection{Immunofluorescence imaging}

HeLa cells stably expressing eGFP-LC3 were seeded on the coverslip with or without CBS. Cells were fixed with cold methanol for $5 \mathrm{~min}$ at $-20{ }^{\circ} \mathrm{C}$. Cells were washed with DPBS three times, followed by incubated with primary antibodies for $3 \mathrm{~h}$ at room temperature. The secondary antibodies and $200 \mathrm{ng}$ $\mathrm{mL}^{-1}$ DAPI were incubated for $1 \mathrm{~h}$ after three times washing with DPBS. The coverslips were washed three times with DPBS and sealed with Mowiol in the dark. Images were captured and analysed using a fluorescence microscope (Olympus DP72).

\subsection{Statistical analysis}

The values obtained in the experiments were expressed as the mean \pm standard deviation (SD) and were analysed by the Student's $t$-test where necessary. All statistical analyses were performed using SPSS 17.0 software and $P<0.05$ was considered statistically significant. 


\section{Results and discussion}

Fig. SI-1 $\uparrow$ displayed the preparation of CBS. For assuring accuracy, even though CBS has been well characterised and reported previously by its provider Prof. Yanfang Su (Tianjin University, China),${ }^{8}$ we also performed LC-MS for CBS sample. The HPLC and total ion chromatography (TIC) MS profiles were listed in Fig. SI-2 and $3, \dagger$ additionally, the chemical profiles of all 15 reported saponins were listed in Table SI-1 and Fig. SI-4. $\dagger$

Fig. 1 depicts the skeleton for saponins in CBS which has been used throughout this study. Saponins from Conyza blinii H.Lév. are biosynthesised through a specific pathway, and hence their structures show remarkable characteristics. For all identified saponins from this species, their aglycones are either $2 \beta, 23$-dihydroxyoleanolic acid or 2 $\beta, 16 \alpha, 23$-trihydroxyoleanolic acid (Fig. 1). The aglycones are substituted with oligosaccharides at $\mathrm{C}-3$ and $\mathrm{C}-28$ to form bisdesmosidic triterpenoidal saponins. The saccharide sequences are highly modular. For the $\alpha$-chains (sugar chain at C-28), they all start by -Ara(2,1)Rha(4,1)-Xyl, which in some cases are further substituted with Api, Ara, Rha, Xyl and Gal. The $\beta$-chain (sugar chain at C-3) is much simpler and has only three combinations, -Glc, -Glc(3,1)Glc, or $-\mathrm{Glc}(3,1)-\mathrm{Xyl}$. $\mathrm{C}-16$ is with either a $-\mathrm{H}$ or $-\mathrm{OH}$ substitution.

To study the effect of CBS on autophagy of cancer cells, we used well-characterised HeLa cell line (human cervical cancer) as a model. We began with the investigation of LC3 (microtubule-associated protein light chain 3). During autophagy, LC3-I, cytoplasmic form of LC3 is converted into lipidated (phosphatidylethanolamine-conjugated) form LC3-II. The latter aggregates onto the membranes of autophagic vacuoles (AVs) and thus is a common diagnostic marker of AP formation. ${ }^{19}$ The abundance of LC3-II and the number of LC3-positive puntca are positively correlated with the amount of intracellular AVs. ${ }^{20}$ In the presence of CBS, the formation of LC3-II increased and showed a clear dose-dependent manner (Fig. 2A). This can

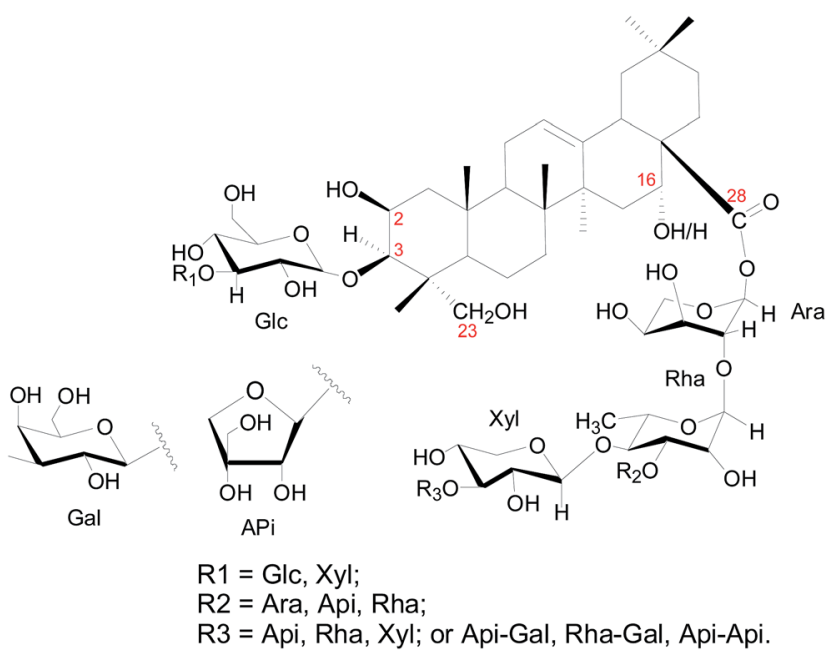

Fig. 1 Structural skeleton of components in CBS. The numbers in red mark the carbon positions. Glc = glucose; Ara = arabinose; Rha = rhamnose; $\mathrm{Xyl}=$ xylose. $\mathrm{Api}=$ apiose; Gal = galactose.

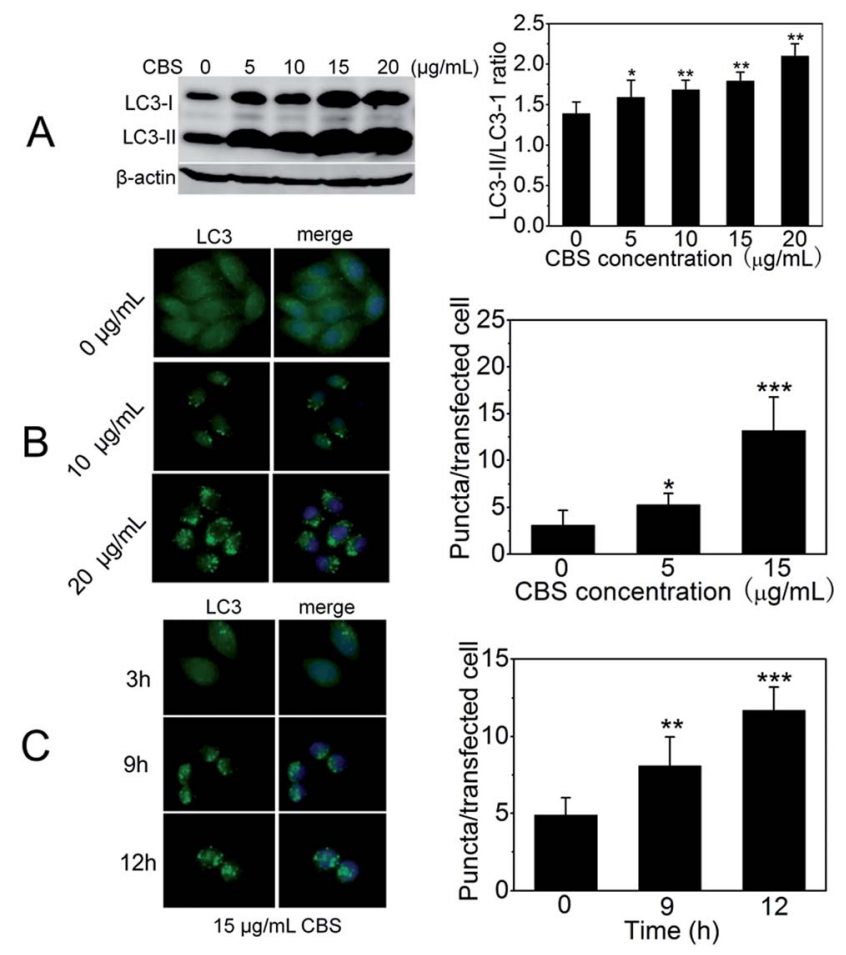

Fig. 2 Autophagic processes are affected by CBS treatment in HeLa cells. (A) The abundance of LC3 of HeLa cells treated with CBS. HeLa cells treated with CBS or vehicle (physiological saline) for $24 \mathrm{~h}$ were examined by Western blotting with LC3 antibody, and $\beta$-actin was used as loading control. (B) Immunofluorescence images showing LC3-II puncta in HeLa cells with different CBS doses (treated for $24 \mathrm{~h}$ ). (C) Immunofluorescence images showing LC3-II puncta in HeLa cells with different incubation times (under $15 \mu \mathrm{g} \mathrm{mL}^{-1} \mathrm{CBS}$ treatment). The mean values and standard deviations are both displayed. $* P<0.05, * * P$ $<0.01, * * * P<0.001$ vs. controls; Student's $t$-test was used.

also be confirmed by immunofluorescence when ectopically expressing eGFP-LC3 plasmid in HeLa cells. As shown in Fig. 2B and C, eGFP-LC3 showed a diffused distribution pattern in control cells, but raised numbers of cytoplasmic LC3-positive puncta were evident in the presence of CBS. This trend was much more apparent when either increasing the CBS dosage (Fig. 2B) or prolonging the incubation time (Fig. 2C). We further proved this observation by TEM images (Fig. 3), which showed that the numbers of AVs (indicated by yellow arrows) were considerably increased in cytoplasm of HeLa cells treated with CBS $(P<0.001)$. When examining all the images taken, double layer membrane APs were hardly seen, while a number of ALs, characteristic of single layer membrane, were commonly observed. Taken together, all these above-mentioned facts support the conclusion that CBS induces the accumulation of AVs in HeLa cells.

The number of AVs observed at any 'snap shot' point is in fact a 'net value' between the rate of their generation and degradation. Thus, AVs accumulation represents either autophagy induction or, alternatively, autophagy suppression. We needed to distinguish these two events. As shown in Fig. 4A, we have measured the abundance of p62, an LC3 binding protein that is degraded by autophagy. ${ }^{22}$ It is one of the best studied 


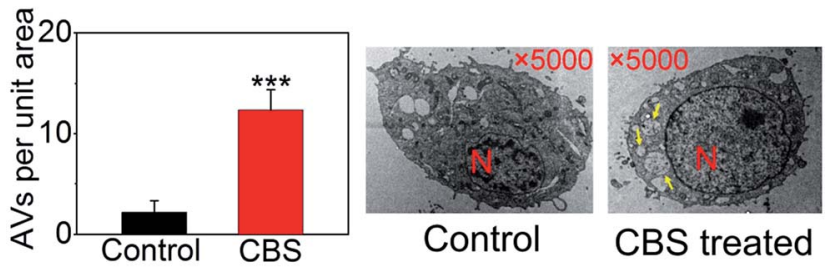

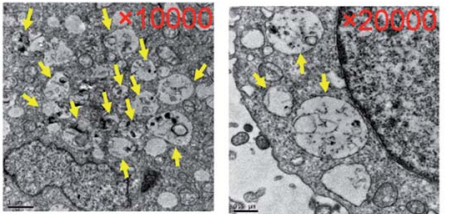

CBS treated

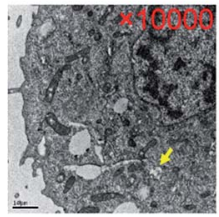

Control
Fig. 3 TEM images depicting ultrastructures of AVs in HeLa cells with or without CBS treatment. HeLa cells treated with $15 \mu \mathrm{g} \mathrm{mL}{ }^{-1} \mathrm{CBS}$ or physiological saline (for the control group) for $12 \mathrm{~h}$ were examined by TEM. Yellow arrows indicate AVs. The mean values and standard deviations are both displayed. ${ }^{* * *} P<0.001$ vs. control; Student's $t$-test was used. substrate of autophagy therefore it reflects autophagic flux. We found that autophagy cargo protein p62 level was strikingly increased in the presence of CBS. Within $15 \mu \mathrm{g} \mathrm{mL}{ }^{-1} \mathrm{CBS}$, p62 reached a plateau at $\sim 12 \mathrm{~h}$. However, the increased level of p62 is possibly owing to two reasons: inhibition of its degradation or up-regulation of its expression. In order to discriminate these two possibilities, we performed an semi-quantitative RT-PCR (reverse transcription polymerase chain reaction) experiment to measure the mRNA of p62 in the presence or absence of CBS, as shown in Fig. 4B. The addition of CBS did not significantly alter the mRNA level of p62 under both conditions: raised CBS dose (Fig. 4B left panel) and extended incubation duration (Fig. 4B right panel). This observation indicates that CBS does not obviously affect the transcription level of p62 and thus ruling out the possibility that CBS increase the expression of p62. Therefore, we can surely conclude that the accumulation of p62 is largely due to the inhibition of its degradation. These data persuasively suggest that CBS functions as a blocker for

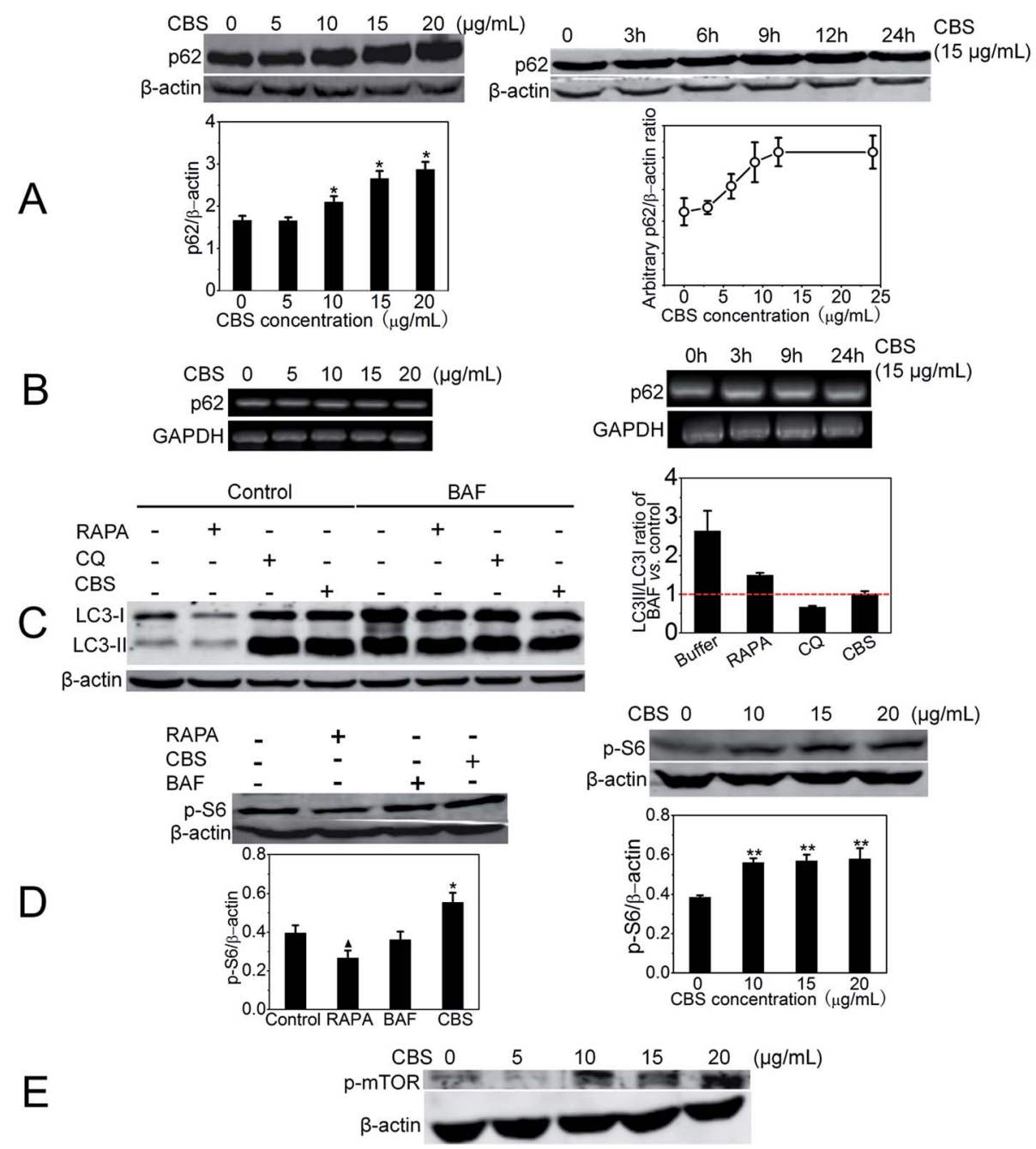

Fig. 4 Autophagic flux in HeLa cells is inhibited by CBS. (A and B) display the abundance of p62 in HeLa cells, which is revealed by Western blot and RT-PCR respectively. (C) Bafilomycin clamp experiment. (D) The abundance of $p$ - $S 6$ revealed by Western blot. HeLa cells were treated with different compounds where necessary for $24 \mathrm{~h}$. (E) The abundance of $\mathrm{p}$-mTOR revealed by Western blot. HeLa cells were treated with different concentrations of CBS for $24 \mathrm{~h}$. The mean values and standard deviations are both displayed. $* P<0.05, * * P<0.01$ vs. controls; Student's $t$-test was used. 
p62 degradation, in other words, it suppresses autophagy. Furthermore, a bafilomycin A1 (BAF) clamp experiment was performed as shown in Fig. 4C. Bafilomycin clamp assay is classically employed in integration with p62 abundance, to identify autophagy modulators. ${ }^{23}$ HeLa cells were treated with water solvent, RAPA, CQ or CBS in the absence or presence of BAF. At $24 \mathrm{~h}$, cells treated with CBS did not demonstrate an increase in LC3-II/LC3-I ratio for BAF-treated cells compared with the control (value around 1), while this LC3-II/LC3-I ratio for CQ and rapamycin (RAPA) were less than and more than 1 respectively. This pattern confirmed that CBS was an autophagy inhibitor rather than an activator. Next, we tested if CBS had effect on p-p70S6 (referred to as p-S6 hereafter) kinase, which was regulated and positively correlated with mTORC1 thus it was deemed as a readout for mammalian target of rapamycin (mTOR) activity. RAPA, a well-known autophagy inducer, was used as a control and it resulted in detectable decrease of p-S6 (lefe panel of Fig. 4D), which was consistent with its mTOR inhibiting activity. BAF did not change p-S6 level. CBS, on the contrary, significantly enhanced the expression of p-S6 in a dosedependent manner (right panel of Fig. 4D). Also, we elicited that CBS up-regulated phospho-mTOR (p-mTOR) level (as shown in Fig. 4E), which indicative of p-mTOR activation by CBS. Both indicate that CBS is an mTOR activator. It is known that mTOR is an important regulator of autophagy and its activation leads to autophagy inhibition. ${ }^{24}$ Targeting mTOR pathway is a new trend for drug discovery. It is noticeable that Astragalus saponins have been shown to down-regulate $\mathrm{mTOR},{ }^{25}$ and panaxatriol saponins have neuroprotective effect through the activation of mTOR pathway. ${ }^{26}$ Taken all, we can reliably conclude that CBS is an autophagy inhibitor. It exerts this effect via a dual targets: mTOR activation and autophagy degradation inhibition.
Next, we sought to know the details of CBS's autophagy inhibition activity. Is this inhibition due to blockage of the fusion of APs with LYs like what BAF and CQ do? The images in Fig. 5A and B showed a clear co-localisation of eGFP-LC3 with two diagnostic makers of LYs, LAMP1 and LAMP2 (indicated by arrows). It seemed that the addition of CBS did not inhibit the fusion of APs with LYs, unlike BAF $^{27}$ and chloroquine (CQ). ${ }^{28}$ This claim can be validated by TEM images, as previously discussed in Fig. 3, we checked all the TEM images and found the most AVs were actually ALs (not all data shown), which indicated that ALs can be formed without any CBS-induced suppression. It is known that CQ is lysosomotropic and inhibits LY acidification, leading to elevated lysosomal pH. BAF is an effective inhibitor of vacuolar-type $\mathrm{H}^{+}$-ATPase, therefore it significantly increases the $\mathrm{pH}$ value of LYs. Both of them deacidify vacuoles, which is the main cause responsible for preventing the fusion between APs and LYs. We then intended to examine if CBS had a similar vacuoles de-acidification effect or not? For this, we used AO (acridine orange) and MDC (monodansylcadaverine) dyes to monitor the acidity of subcellular compartments. $\mathrm{AO}$ is an acidotropic fluorochrome which changes to orange-red fluorescence at lower $\mathrm{pH}$ values. ${ }^{29}$ From Fig. 5B, it can be seen CBS caused an accumulation of acidic vesicles, alike RAPA. While BAF almost de-acidified cells as evidenced by the fact that there was hardly orange-red fluorescence observed. We also used flow cytometry to quantify the fluorescence intensity. As shown in Fig. 5B, BAF-treated HeLa cells emitted least red fluorescence than the other three groups, while for CBS-treated cells, their brightness of fluorostaining slightly increased than the controls. This result is consistent with the fluorescence microscopic images and implies that CBS does not de-acidify the LYs or fused ALs. MDC was originally

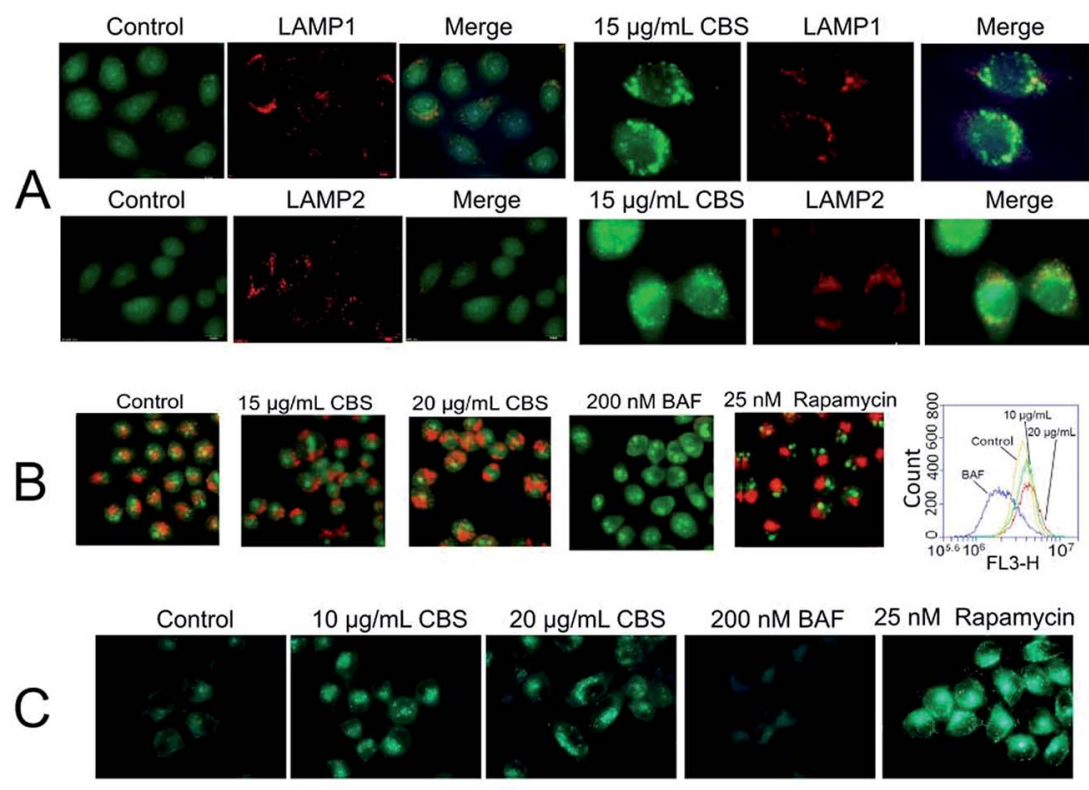

Fig. 5 CBS does not significantly prevent autophagolysosome formation and alter the pH values of autophagic vacuoles. (A) Immunofluorescent images depicting co-localisation of LC3-II with LAMP1 and LAMP2. (B) Fluorescence images and flow cytometry profiles of HeLa cells treated with different agents after AO staining. (C) Fluorescence images of HeLa cells after MDC staining. HeLa cells were treated with CBS or other compounds where necessary for $12 \mathrm{~h}$. 
proposed to be a specific AP indicator but was later demonstrated to have higher affinity for LYs. ${ }^{30,31}$ It is now thought that MDC preferentially labels acidic compartments. As shown in Fig. 5C, MDC fluorescent intensity was significantly enhanced in CBS-treated cells, whereas dramatically decreased in BAFtreated cells. This agreed well with the AO staining experiment and further suggested that the increased AVs in CBStreated cells were acidic ALs. Detailed molecular mechanisms adopted by CBS to inhibit autophagy require further exploration.

Autophagy is key process for cell homeostasis, and it is believed to be highly associated with cancers. ${ }^{32,33}$ We are interested in hunting autophagy modulators for cancer treatment. We have confirmed that CBS is an autophagy inhibitor. Interestingly, it is not like CQ and BAF, both of which impede the incorporation of APs and LYs, CBS seems not to perceptibly hider the fusion process, but inhibits the autophagic degradation. In addition, CBS is found to be an mTOR activator. Autophagy is a rather dynamic process composed of five successive steps: initiation, elongation, closure, maturation and degradation. ${ }^{21}$ In order words, CBS blocks the degradation and activates the initiation processes hence it is a dual-targeting inhibitor. There is an upsurge for discovery of synthetic and natural autophagy inhibitors for cancer therapy. Martine, liensinine and Lys05 represents fine newly discovered autophagy inhibitors. ${ }^{34-36}$ It has been reported that some autophagy inhibiting compounds are capable of sensitising chemotherapy. ${ }^{37,38}$ It is known that, in the process of distal autophagy inhibition, the accumulation of dysfunctional AVs and eventual bursting of lysosomes lead to cell death through apoptosis or necrosis. Moreover, Yang et al. have demonstrated that genetically or pharmacologically inhibiting autophagy incurs robust tumour growth suppression for certain types of cancers, which results from reactive oxygen species increase and DNA damage generation. ${ }^{39,40}$ Therefore, inhibition of autophagy is beneficial for anticancer therapies. ${ }^{\mathbf{4 1}}$ There are a list of clinical trials underway (https://clinicaltrials.gov/) to evaluate whether the inhibition of autophagy has synergistic effects in a variety of human cancers; for example, chloroquine as an anti-autophagy drug in stage IV small cell lung cancer (SCLC) patients. Unfortunately, few autophagy inhibitors have been approved to be used clinically for treating cancers up to now. Thus, novel autophagy inhibitor with a lower toxicity and a better therapeutic index is urgently needed. Very few saponins have been reported as autophagy modulators and most of them are autophagy inducers, including soybean B-group triterpenoid saponins, akebia saponin PA and timosaponin A-III. ${ }^{\mathbf{4 2 - 4 4}}$ It is rare for saponin to act as an autophagy inhibitor against cancer and CBS is an extraordinary example in this regard. Strikingly, CBS, when being used solely, is found to have potent anti-cancer activity both in vitro and in vivo by our group previously, which is on account of its NF- $\kappa$ B inhibition. ${ }^{7,8}$ The distal autophagy inhibition of CBS revealed in current study seems to be another cause for such activity, which pushes one step forward for its development as an anti-cancer agent.

Botanical/herbal drugs have been traditionally used and nowadays they are getting increasingly important. EMA launched 'Traditional Herbal Medicinal Product Directive 24/ EC/2004' (THMPD) long back to the year 2004, and USFDA approved the first orally taken botanical drug Crofelemer in 2012. These efforts have initialised the idea to turn 'traditional' and/or 'experience-based' botanical drugs to 'modern' and 'scientifically-proved' ones. This also encourages us to study and develop evidence-based botanical drugs. In current study, we have not yet revealed the molecular targets for CBS's autophagic inhibition effect, which we think is certainly beyond the scope of this paper and will be further elucidated in futuristic work. To conclude, we have found that CBS is an effective blocker of autophagy for HeLa cells and it has dual targets to modulate autophagic process. This is probably on account of its anti-cancer activity. Additionally, our novel finding expands the activity of saponins as autophagy inhibitors. ${ }^{45-48}$

\section{Author contributions}

Profs. Long Ma designed the experiments. Haiyan Liu, Chenxi $\mathrm{Hu}$, Nana Sun carried out most experiments and analysed the data. Drs Aipo Diao, Yuyin Li, Shuli Man, and Zhenxing Liu gave some valuable suggestions and technical supports. Long $\mathrm{Ma}$ wrote the manuscript.

\section{Conflict of interest}

The authors wish to confirm that there is no competing financial interests.

\section{Acknowledgements}

This work is supported by National Natural Science Foundation of China (No. 81503086, 21672161, 81673647), a starting funding (No. 20140520) from Tianjin University of Science \& Technology (TUST), a research funding of "1000 Talents Plan" of Tianjin and a funding from Foundation of Key Laboratory of Industrial Fermentation Microbiology of Ministry of Education and Tianjin Key Lab of Industrial Microbiology (No. 2015IM106). We thank Prof. Yanfang Su (School of Pharmaceutical Science and Technology, Tianjin University, China) for making and providing the CBS sample.

\section{References}

1 H. Deng, L. Ma, N. Bandaranayaka, Z. Qin, G. Mann, K. Kyeremeh, Y. Yu, T. Shepherd, J. H. Naismith and D. O'Hagan, ChemBioChem, 2014, 15, 364-368.

2 J. Clardy and C. Walsh, Nature, 2004, 432, 829-837.

3 L. Ma, A. Bartholome, M. H. Tong, Z. Qin, Y. Yu, T. Shepherd, K. Kyeremeh, H. Deng and D. O'Hagan, Chem. Sci., 2015, 6, 1414-1419.

4 L. Ma, Y. Li, L. Meng, H. Deng, Y. Li, Q. Zhang and A. Diao, RSC Adv., 2016, 6, 27047-27051.

5 D. J. Newman and G. M. Cragg, J. Nat. Prod., 2016, 79, 629661.

6 L. Ma and A. Diao, Anti-Cancer Agents Med. Chem., 2015, 15, 298-306. 
7 Chinese Pharmacopoeia Commission, Pharmacopoeia of the People's Republic of China, People's Medical Publishing House, Beijing, China, 2010, vol. I, p. 201.

8 X. Qiao, X. Zhang, M. Ye, Y. Su, J. Dong, J. Han, J. Yin and D. Guo, Rapid Commun. Mass Spectrom., 2010, 24, 3340-3350. 9 Y. Su, D. Guo, H. Guo, J. Liu, J. Zheng, K. Koike and T. Nikaido, J. Nat. Prod., 2001, 64, 32-36.

10 Y. Su, K. Koike, D. Guo, T. Satou, J. Liu, J. Zheng and T. Nikaido, Tetrahedron, 2001, 57, 6721-6726.

11 Y. Su, K. Koike, T. Nikaido, J. Liu, J. Zheng and D. Guo, J. Nat. Prod., 2003, 66, 1593-1599.

12 L. Ma and J. Liu, J. Ethnopharmacol., 2014, 158, 358-363.

13 L. Ma, H. Liu, L. Meng, P. Qin, B. Zhang, Y. Li, S. Man, Z. Liu, Z. Liu and A. Diao, RSC Adv., 2017, 7, 3408-3412.

14 L. Ma, H. Liu, P. Qin, C. Hu, S. Man, Y. Li, Z. Liu, Z. Liu and A. Diao, Biochem. Biophys. Res. Commun., 2017, 483, 779-785.

15 N. Mizushima, T. Yoshimori and B. Levine, Cell, 2010, 140, 313-326.

16 N. Mizushima, B. Levine, A. M. Cuervo and D. J. Klionsky, Nature, 2008, 451, 1069-1075.

17 K. Degenhardt, R. Mathew, B. Beaudoin, K. Bray, D. Anderson, G. Chen, C. Mukherjee, Y. Shi, C. Gélinas and Y. Fan, Cancer Cell, 2006, 10, 51-64.

18 R. Mathew and E. White, Curr. Opin. Genet. Dev., 2011, 21, 113-119.

19 Y. Kabeya, N. Mizushima, T. Ueno, A. Yamamoto, T. Kirisako, T. Noda, E. Kominami, Y. Ohsumi and T. Yoshimori, EMBO J., 2000, 19, 5720-5728.

20 N. Mizushima, Int. J. Biochem. Cell Biol., 2004, 36, 2491-2502. 21 A. C. Kimmelman, Genes Dev., 2011, 25, 1999-2010.

22 R. Mathew, C. M. Karp, B. Beaudoin, N. Vuong, G. Chen, H.-Y. Chen, K. Bray, A. Reddy, G. Bhanot and C. Gelinas, Cell, 2009, 137, 1062-1075.

23 S. Murugan and R. K. Amaravadi, in Tumor Microenvironment: Study Protocols, ed. C. Koumenis, M. L. Coussens, A. Giaccia and E. Hammond, Springer International Publishing, Cham, 2016, pp. 145-166.

24 B. Ravikumar, C. Vacher, Z. Berger, J. E. Davies, S. Luo, L. G. Oroz, F. Scaravilli, D. F. Easton, R. Duden, C. J. O'Kane and D. C. Rubinsztein, Nat. Genet., 2004, 36, 585-595.

25 K. K. Auyeung, N. L. Mok, C. M. Wong, C. H. Cho and J. K. Ko, Int. J. Mol. Med., 2010, 26, 341-349.

26 C. Zhang, C. Li, S. Chen, Z. Li, L. Ma, X. Jia, K. Wang, J. Bao, Y. Liang, M. Chen, P. Li, H. Su, S. M. Y. Lee, K. Liu, J.-B. Wan and C. He, Sci. Rep., 2017, 7, 41082.
27 A. Yamamoto, Y. Tagawa, T. Yoshimori, Y. Moriyama, R. Masaki and Y. Tashiro, Cell. Struct. Funct., 1998, 23, 33-42. 28 V. R. Solomon and H. Lee, Eur. J. Pharmacol., 2009, 625, 220233.

29 C. L. Vazquez and M. I. Colombo, Methods Enzymol., 2009, 452, 85-95.

30 A. Biederbick, H. Kern and H. Elsässer, Eur. J. Cell Biol., 1995, 66, 3-14.

31 E. T. Bampton, C. G. Goemans, D. Niranjan, N. Mizushima and A. M. Tolkovsky, Autophagy, 2005, 1, 23-36.

32 Y. Kondo and S. Kondo, Autophagy, 2006, 2, 85-90.

33 N. Mizushima and T. Yoshimori, Autophagy, 2007, 3, 542545.

34 Z. Wang, J. Zhang, Y. Wang, R. Xing, C. Yi, H. Zhu, X. Chen, J. Guo, W. Guo, W. Li, L. Wu, Y. Lu and S. Liu, Carcinogenesis, 2013, 34, 128-138.

35 J. Zhou, G. Li, Y. Zheng, H.-M. Shen, X. Hu, Q.-L. Ming, C. Huang, P. Li and N. Gao, Autophagy, 2015, 11, 1259-1279.

36 Q. McAfee, Z. Zhang, A. Samanta, S. M. Levi, X.-H. Ma, S. Piao, J. P. Lynch, T. Uehara, A. R. Sepulveda and L. E. Davis, Proc. Natl. Acad. Sci. U. S. A., 2012, 109, 82538258.

37 Z. J. Yang, C. E. Chee, S. Huang and F. A. Sinicrope, Mol. Cancer Ther., 2011, 10, 1533-1541.

38 C. Wang, Q. Hu and H.-M. Shen, Pharmacol. Res., 2016, 105, 164-175.

39 P. Boya and G. Kroemer, Oncogene, 2008, 27, 6434-6451.

40 S. Yang, X. Wang, G. Contino, M. Liesa, E. Sahin, H. Ying, A. Bause, Y. Li, J. M. Stommel and G. Dell'Antonio, Genes Dev., 2011, 25, 717-729.

41 Y. Koike, T. Toide, A. Sutoh, M. Murakami and K. Oda, Nat. Rev. Cancer, 2005, 5, 726-734.

42 A. A. Ellington, M. Berhow and K. W. Singletary, Carcinogenesis, 2005, 26, 159-167.

43 M.-Y. Xu, D. H. Lee, E. J. Joo, K. H. Son and Y. S. Kim, Food Chem. Toxicol., 2013, 59, 703-708.

44 L.-K. Sy, S.-C. Yan, C.-N. Lok, R. Y. K. Man and C.-M. Che, Cancer Res., 2008, 68, 10229-10237.

45 B. Y. Law, S. W. Mok, A. G. Wu, C. W. Lam, M. X. Yu and V. K. Wong, Molecules, 2015, 21, 359.

46 P. Qiu, S. Man, H. Yang, Y. Liu, Z. Liu, L. Ma, P. Yu and W. Gao, RSC Adv., 2016, 6, 115029-115038.

47 J. Liu, Z. Liu, S. Man, H. Chai, L. Ma and W. Gao, RSC Adv., 2016, 6, 92330-92334.

48 S. Man, J. Li, P. Qiu, J. Liu, Z. Liu, L. Ma and W. Gao, Mol. Carcinog., 2017, 56, 1405-1413. 